\title{
Molecular Carbon Chains and Rings in TMC-1
}

\author{
David Fossé ${ }^{1,2}$, José Cernicharo ${ }^{1}$, Maryvonne Gerin ${ }^{2}$, and Pierre Cox ${ }^{3}$ \\ ${ }^{1}$ Depto Física Molecular, I.E.M., C.S.I.C., Serrano 121, E-28006 Madrid, Spain \\ ${ }^{2}$ L.R.A., Observatoire de Paris \& Ecole Normale Supérieure, 24 rue Lhomond, F-75231 \\ Paris Cedex 05, France \\ 3 I.A.S., Université Paris-Sud, bâtiment 121, F-91405 Orsay, France
}

Received ** 2000; $\quad$ accepted $15 / 12 / 2000$

\footnotetext{
${ }^{1}$ e-mail: David.Fosse@lra.ens.fr
} 


\begin{abstract}
We present mapping results in several rotational transitions of $\mathrm{HC}_{3} \mathrm{~N}$, $\mathrm{C}_{6} \mathrm{H}$, both cyclic and linear $\mathrm{C}_{3} \mathrm{H}_{2}$ and $\mathrm{C}_{3} \mathrm{H}$, towards the cyanopolyyne peak of the filamentary dense cloud TMC-1 using the IRAM $30 \mathrm{~m}$ and MPIfR $100 \mathrm{~m}$ telescopes. The spatial distribution of the cumulene carbon chain propadienylidene $\mathrm{H}_{2} \mathrm{CCC}$ (hereafter $l-\mathrm{C}_{3} \mathrm{H}_{2}$ ) is found to deviate significantly from the distributions of the cyclic isomer $c-\mathrm{C}_{3} \mathrm{H}_{2}, \mathrm{HC}_{3} \mathrm{~N}$, and $\mathrm{C}_{6} \mathrm{H}$ which in turn look very similar. The cyclic over linear abundance ratio of $\mathrm{C}_{3} \mathrm{H}_{2}$ increases by a factor of 3 across the filament, with a value of 28 at the cyanopolyyne peak. This abundance ratio is an order of magnitude larger than the range ( 3 to 5 ) we observed in the diffuse interstellar medium. The cyclic over linear abundance ratio of $\mathrm{C}_{3} \mathrm{H}$ also varies by $\sim 2.5$ in TMC-1, reaching a maximum value (13) close to the cyanopolyyne peak. These behaviors might be related to competitive processes between ion-neutral and neutral-neutral reactions for cyclic and linear species.
\end{abstract}

Subject headings: ISM: abundances — ISM: individual (TMC-1) — ISM: molecules — molecular processes 


\section{Introduction}

Among the molecules discovered in the interstellar medium, $\mathrm{C}_{3} \mathrm{H}_{2}$ and $\mathrm{C}_{3} \mathrm{H}$ are of peculiar interest for astrochemistry since both are observed in two isomeric forms: cyclic and linear. The ring molecule cyclopropenylidene (hereafter $c-\mathrm{C}_{3} \mathrm{H}_{2}$ ) was discovered in 1985 (Thaddeus, Vrtilek, \& Gottlieb) and has immediatly retained attention due to its ubiquity in the galaxy (e.g., Matthews and Irvine 1985). One of its linear counterparts, the propadienylidene $\left(l-\mathrm{C}_{3} \mathrm{H}_{2}\right)$, was discovered in TMC-1 by Cernicharo et al. (1991). The two isomeric shapes of $\mathrm{C}_{3} \mathrm{H}$, cyclopropynylidyne $\left(c-\mathrm{C}_{3} \mathrm{H}\right)$ and propynylidyne $\left(l-\mathrm{C}_{3} \mathrm{H}\right)$, were also detected in TMC-1 (Yamamoto et al. 1987; Thaddeus et al. 1985).

This variety of isomers for the same species raises the question of their formation. In particular, are rings and chains formed from the same progenitors and involved in the same reactions networks? Furthermore these isomers can be used to probe interstellar chemistry models which include heavy interstellar molecules such as cumulene carbenes (Bettens and Herbst 1996, 1997; see also Millar, Leung, and Herbst 1987). Previous observations by Cernicharo et al. (1999) have shown that the cyclic over linear abundance ratio of $\mathrm{C}_{3} \mathrm{H}_{2}$ (hereafter $\mathrm{R}_{2}$ ) in the diffuse medium along the line of sight toward the continuum sources W51E1/E2, W51D and W49 is one order of magnitude smaller than its value in TMC- 1. In a recent study, Turner, Herbst, \& Terzieva (2000) have compared measurements in three translucent clouds and two dense clouds (TMC-1 and L183) of the $\mathrm{C}_{3} \mathrm{H}$ cyclic over linear abundance ratio $\left(R_{1}\right)$ and $R_{2}$ ratio - and column densities of 6 other hydrocarbons - to a modified version of the New Standard Model of chemistry (Lee et al. 1996). They found small variation of $R_{1}$ from source to source, and a systematic higher $R_{2}$ ratio with largest the values found in the two dark clouds. Also, it has been suggested that cyclic and linear isomers of a same compound could have different behavior in neutral-neutral and ion-neutral reactions (see, for example, Adams \& Smith 1987; Maluendes, McLean, \& Herbst 1993; Kaiser et al. 1997, 1999). The cyclic over linear abundance ratio of molecules like $\mathrm{C}_{3} \mathrm{H}_{2}$ and $\mathrm{C}_{3} \mathrm{H}$ could then be used as a tool to investigate physical conditions in several media, from cold dark cloud to warmer and lower density media.

In order to investigate these questions, we mapped in detail the region around the cyanopolyyne peak (hereafter CP) in TMC- 1 in both $l-\mathrm{C}_{3} \mathrm{H}_{2}$ and $c-\mathrm{C}_{3} \mathrm{H}_{2}$, and observed at

selected positions the two isomeric forms of $\mathrm{C}_{3} \mathrm{H} . \mathrm{C}_{6} \mathrm{H}$ and $\mathrm{HC}_{3} \mathrm{~N}$ have also been observed for comparison purpose. 


\section{Observations}

The $\mathrm{C}_{3} \mathrm{H}_{2}$ and $\mathrm{C}_{6} \mathrm{H}$ observations were made in 1990 and 1992 with the 100 -meter telescope of the Max-Planck Institut für Radioastronomie at Effelsberg (Germany). We observed the $1_{10}-1_{01}$ transitions of $c-\mathrm{C}_{3} \mathrm{H}_{2}$ at $18343.145 \mathrm{MHz}$, and simultaneously those of $\mathrm{C}_{6} \mathrm{H}^{2} \Pi_{3 / 2} \mathrm{~J}=15 / 2-13 / 2$ near $20794 \mathrm{MHz}$ and $l-\mathrm{C}_{3} \mathrm{H}_{2} 1_{01}-0_{00}$ at $20792.590 \mathrm{MHz}$. The antenna beamsize at 18.3 and $20.7 \mathrm{GHz}$ are of $54^{\prime \prime}$ and $48^{\prime \prime}$, respectively. We used the 1024 channel autocorrelator to achieve a resolution of $0.05 \mathrm{~km} \mathrm{~s}^{-1}$ at $18.3 \mathrm{GHz}$ and $0.09 \mathrm{~km} \mathrm{~s}^{-1}$ at $20.7 \mathrm{GHz}$, and the data were taken by position switching the telescope.

The $\mathrm{HC}_{3} \mathrm{~N}$ and $\mathrm{C}_{3} \mathrm{H}$ observations were done in 1995 and 1999 with the IRAM 30-meter telescope at Pico Veleta (Spain). We observed the $l-\mathrm{C}_{3} \mathrm{H}^{2} \Pi_{3 / 2} \mathrm{~J}=9 / 2-7 / 2$ transition near $98 \mathrm{GHz}$ and at the same time the transition of $c-\mathrm{C}_{3} \mathrm{H} 2_{12}-1_{11}$ near $91.5 \mathrm{GHz}$. The observations were made in frequency-switching mode. The autocorrelator was used as a spectral instrument with a velocity resolutions of $0.12 \mathrm{kms}^{-1}$. The half-power beamwidth (HPBW) and main-beam efficiency are 26 " and 0.75 for $l-\mathrm{C}_{3} \mathrm{H}$ and 27 " and 0.78 for $c-\mathrm{C}_{3} \mathrm{H}$. System temperatures were in the range 90-130K. For $\mathrm{HC}_{3} \mathrm{~N}$, observed at $90979.023 \mathrm{MHz}$ $(\mathrm{J}=10-9)$, the HPBW is $26 "$. Pointing and calibration were monitored by regularly observing planets and quasars for both telescopes. Sample spectra are shown in Fig.1 and line parameters for selected positions are given in Table 1.

\section{Results}

\section{1. $\mathrm{C}_{3} \mathrm{H}_{2}$ isomers and $\mathrm{C}_{6} \mathrm{H}$}

The mapping results of this study are shown in Fig. 2. The distributions towards the $\mathrm{CP}$ in TMC-1 of $c-\mathrm{C}_{3} \mathrm{H}_{2}$ and $\mathrm{l}_{3} \mathrm{C}_{2}$ are compared to that of $\mathrm{C}_{6} \mathrm{H}$ in Fig. $2 \mathrm{a}$ and $\mathrm{b}$, respectively. Fig. 2c shows the distributions of $\mathrm{C}_{6} \mathrm{H}$ and $\mathrm{HC}_{3} \mathrm{~N}$. The spatial distributions of $l-\mathrm{C}_{3} \mathrm{H}_{2}$ and $c-\mathrm{C}_{3} \mathrm{H}_{2}$ (Fig. 2d) are clearly different, whereas the emissions of $c-\mathrm{C}_{3} \mathrm{H}_{2}, \mathrm{C}_{6} \mathrm{H}$ and $\mathrm{HC}_{3} \mathrm{~N}$ have comparable distributions. The emission of $l-\mathrm{C}_{3} \mathrm{H}_{2}$ is shifted toward the west by approximatively 40 " with respect to $c-\mathrm{C}_{3} \mathrm{H}_{2}$ or $\mathrm{C}_{6} \mathrm{H}$ - note that, since $\mathrm{C}_{6} \mathrm{H}$ and $l-\mathrm{C}_{3} \mathrm{H}_{2}$ were observed in the same bandwidth, this shift cannot be due to pointing errors during the observations.

In order to derive relative abundances, we first computed relations between the column-density and the observed line intensity valid for uniform physical conditions, i.e. $\mathrm{n}\left(\mathrm{H}_{2}\right)=3 \times 10^{4} \mathrm{~cm}^{-3}$ and $\mathrm{T}_{K}=10 \mathrm{~K}$ in TMC-1 (Cernicharo \& Guélin 1987). From a statistical equilibrium calculation using collisional excitation rates for $c-\mathrm{C}_{3} \mathrm{H}_{2}$ from Avery 
\& Green (1989), and assuming an ortho/para ratio of 3 , we find: $N\left(c-\mathrm{C}_{3} \mathrm{H}_{2}\right)\left[\mathrm{cm}^{-2}\right]=$ $2.2 \times 10^{13} \int T_{m b} d v\left[\mathrm{~K} \cdot \mathrm{km} \cdot \mathrm{s}^{-1}\right]$. This linear relationship indicates that the $1_{10}-1_{01}$ transition of $c-\mathrm{C}_{3} \mathrm{H}_{2}$ is optically thin in TMC-1; it is in good agreement with the work of Cox et al. (1989). Madden et al. (1986), from observations of the isotopic $c-\mathrm{C}_{2}{ }^{13} \mathrm{CH}_{2}$ in TMC-1, derive an optical depth ranging from 5.6 to 6.8 for the $2_{12}-1_{01}$ transition of $c-\mathrm{C}_{3} \mathrm{H}_{2}$ at 85 GHz. This result is in agreement with ours since the $2_{12}-1_{01}$ line has a larger opacity than the $1_{10}-1_{01}$ transition for dark cloud physical conditions. Indeed, with our code, we are able to reproduce the results of Madden et al. at $85 \mathrm{GHz}$, the $1_{10}-1_{01}$ transition still being thin or marginally saturated $(\tau \leq 1.5)$. For $l-\mathrm{C}_{3} \mathrm{H}_{2}$ and $\mathrm{C}_{6} \mathrm{H}$ there are no collisional cross sections available. We have estimated cross sections from those of $\mathrm{H}_{2} \mathrm{CO}$ (calculated by Green et al. 1978) and of $\mathrm{HC}_{3} \mathrm{~N}$ (Green \& Chapman 1978) - see Cernicharo et al. (1999). This gives: $N\left(l-\mathrm{C}_{3} \mathrm{H}_{2}\right)\left[\mathrm{cm}^{-2}\right]=2.2 \times 10^{13} \int T_{m b} d v\left[\mathrm{~K} \cdot \mathrm{km} \cdot \mathrm{s}^{-1}\right]$ and $N\left(\mathrm{C}_{6} \mathrm{H}\right)\left[\mathrm{cm}^{-2}\right]=$ $5.2 \times 10^{13} \int T_{m b} d v\left[\mathrm{~K} \cdot \mathrm{km} \cdot \mathrm{s}^{-1}\right]$. Note that for $\mathrm{C}_{6} \mathrm{H}, \int T_{m b} d v$ is the total integrated intensity summed over the four components of the hyperfine structure. These relationships are in good agreement with previous works (Cernicharo et al. 1991; Bell et al. 1999).

Comparing two positions separated by 40" (which corresponds to $0.02 \mathrm{pc}$ for the adopted distance of 100 pc to TMC-1 - Cernicharo \& Guélin 1987), we derive the following column density: at the $\mathrm{CP}(0,0), N\left(\mathrm{C}_{6} \mathrm{H}\right)=8.3 \times 10^{12} \mathrm{~cm}^{-2}, N\left(c-\mathrm{C}_{3} \mathrm{H}_{2}\right)=5.8 \times 10^{13} \mathrm{~cm}^{-2}$, $N\left(l-\mathrm{C}_{3} \mathrm{H}_{2}\right)=2.1 \times 10^{12} \mathrm{~cm}^{-2}\left(\mathrm{R}_{2}=28 \pm 6\right)$; and at the edge of the TMC-1 filament $(-40,0)$, $N\left(\mathrm{C}_{6} \mathrm{H}\right)=4.7 \times 10^{12} \mathrm{~cm}^{-2}, N\left(c-\mathrm{C}_{3} \mathrm{H}_{2}\right)=2.8 \times 10^{13} \mathrm{~cm}^{-2}, N\left(l-\mathrm{C}_{3} \mathrm{H}_{2}\right)=3.2 \times 10^{12} \mathrm{~cm}^{-2}$ $\left(\mathrm{R}_{2}=10 \pm 3\right)$. Between these two positions, the cyclic over linear abundance ratio changes by a factor of $\sim 3$. This variation cannot be an artefact caused by calibration errors. Indeed, while the calculated $\mathrm{C}_{6} \mathrm{H}$ over $l-\mathrm{C}_{3} \mathrm{H}_{2}$ column density ratio varies from 1.5 to 4 , the $c-\mathrm{C}_{3} \mathrm{H}_{2}$ over $\mathrm{C}_{6} \mathrm{H}$ column density ratio remains constant $(\simeq 6.5)$. Since the former ratio is unaffected by calibration and pointing errors (lines are observed in the same bandwidth), the observed variation of $\mathrm{R}_{2}$ must be real. This result suggests that chemical gradients are present in TMC-1 on scales smaller than 0.02 pc.

To investigate further the spatial variations of molecular abundances across the TMC-1 filament, we averaged the spectra along the six different rows shown in Fig.2. Results are summarized in Fig.3: $\mathrm{R}_{2}$ ranges from 12 to 37 and clearly increases from the west to the east of TMC-1. Note that a constant density of $\mathrm{n}\left(\mathrm{H}_{2}\right)=3 \times 10^{4} \mathrm{~cm}^{-3}$ has been adopted. If we use instead $\mathrm{H}_{2}$ densities derived by Pratap et al. (1997) from an analysis of the $\mathrm{HC}_{3} \mathrm{~N}$ transitions while keeping the same kinetic temperature $(10 \mathrm{~K})$, the values of $\mathrm{R}_{2}$ are then lowered by 20 to $40 \%$ but the west to east variation of $\mathrm{R}_{2}$ remains. The rise of the cyclic over linear abundance ratio of the $\mathrm{C}_{3} \mathrm{H}_{2}$ isomers at a spatial scale of $2 \operatorname{arcmin}(0.06 \mathrm{pc})$ is therefore a firm result. 


\section{2. $\mathrm{C}_{3} \mathrm{H}$ isomers}

As $\mathrm{C}_{3} \mathrm{H}$ is thought to be formed by the same reaction as $\mathrm{C}_{3} \mathrm{H}_{2}$, i.e. by the dissociative recombination of $\mathrm{C}_{3} \mathrm{H}_{3}^{+}$(Adams \& Smith 1987), it is interesting to compare $N\left(c-\mathrm{C}_{3} \mathrm{H}\right) / N(l-$ $\mathrm{C}_{3} \mathrm{H}$ ) (hereafter $\mathrm{R}_{1}$ ) with $\mathrm{R}_{2}$. What is the value of $\mathrm{R}_{1}$ and does it vary across the filament similarly to $\mathrm{R}_{2}$ ? In order to answer these questions we observed $c-\mathrm{C}_{3} \mathrm{H}$ and $l-\mathrm{C}_{3} \mathrm{H}$ at 13 positions along two strips close to the CP. One strip crosses the filament from offset positions $(60 ", 60 ")$ to $(-60 ",-60 ")$, the other from $(10,-70 ")$ to $(-70 ", 10 ")$. We also observed the $c-\mathrm{C}_{3} \mathrm{H}_{2}$ and the $l-\mathrm{C}_{3} \mathrm{H}_{2}$ peaks. Where possible, we analyzed the data with the HFS method of CLASS (a software developed by the GILDAS working group). This method provides the total optical depth, the average linewidth and the brightness temperature of a line with hyperfine structure. A reliable estimate of the excitation temperature could be obtained for several points. We found that $3 \mathrm{~K} \leq \mathrm{T}_{e x} \leq 3.8 \mathrm{~K}$ for $c-\mathrm{C}_{3} \mathrm{H}$ (in excellent agreement with Mangum \& Wootten 1990), and $4.9 \mathrm{~K} \leq \mathrm{T}_{e x} \leq 6.7 \mathrm{~K}$ for $l-\mathrm{C}_{3} \mathrm{H}$. In the following, we adopt $T_{\mathrm{ex}}=3.5 \mathrm{~K}$ for $c-\mathrm{C}_{3} \mathrm{H}$ and $T_{\mathrm{ex}}=5.5 \mathrm{~K}$ for the linear isomer.

Calculations of the column densities were done using the classical formula with $\mu$ $=2.4 \mathrm{D}$ (Yamamoto et al. 1987) for $c-\mathrm{C}_{3} \mathrm{H}$ and $\mu=3.1 \mathrm{D}$ (Green 1980) for $l-\mathrm{C}_{3} \mathrm{H}$. For each observed point, we computed the average of the total column densities by using each hyperfine transition weighted by the inverse of the square of the error. The resulting column densities and the values for $R_{1}$ are given in Table 2. Comparing with the values of $R_{2}$ in Fig.3, we see that: 1) With a value of 12 at the $C P, R_{1}$ is smaller than $\left.R_{2} ; 2\right)$ As $R_{2}$ does, $\mathrm{R}_{1}$ shows variations across the filament (by a factor of 2.5 ), mainly due to variations of $N\left(c-\mathrm{C}_{3} \mathrm{H}\right)$.

\section{Discussion}

The observations described above underscore the differences in the distribution and relative abundances of the cyclic and linear forms of $\mathrm{C}_{3} \mathrm{H}_{2}$ and $\mathrm{C}_{3} \mathrm{H}$ in the molecular filament TMC-1. In the following, we study the origin of this behavior and suggest that it is driven by competitive processes between ion-neutral and neutral-neutral reactions.

\subsection{Steady-state calculation}

We assume here, as it has been proposed (see, for example, Adams \& Smith 1987; Maluendes, McLean \& Herbst 1993), that cyclic $\mathrm{C}_{3} \mathrm{H}_{2}$ and $\mathrm{C}_{3} \mathrm{H}$ are both formed in the dissociative recombination of c- $\mathrm{C}_{3} \mathrm{H}_{3}^{+}$, while linear $\mathrm{C}_{3} \mathrm{H}_{2}$ and $\mathrm{C}_{3} \mathrm{H}$ result from $\mathrm{H}_{2} \mathrm{C}_{3} \mathrm{H}^{+}$. We 
also assume that the $\mathrm{C}_{3} \mathrm{H}^{+}+\mathrm{H}_{2}$ association reaction is the dominant formation mechanism of $\mathrm{C}_{3} \mathrm{H}_{3}^{+}$, which is produced in equal amounts in cyclic and linear forms.

At steady state, the chemical kinetic equation for the abundance of $c-\mathrm{C}_{3} \mathrm{H}_{2}$ - that is, $\mathrm{x}\left(c-\mathrm{C}_{3} \mathrm{H}_{2}\right)$ - is:

$$
\mathrm{dx}\left(c-\mathrm{C}_{3} \mathrm{H}_{2}\right) / \mathrm{dt}={ }^{2} \mathrm{~b}_{c} \mathrm{k}_{c}^{r} \mathrm{x}\left(\mathrm{c}-\mathrm{C}_{3} \mathrm{H}_{3}^{+}\right) \mathrm{x}_{e}-{ }^{2} \mathrm{~K}_{c}^{d} \mathrm{x}\left(\mathrm{c}-\mathrm{C}_{3} \mathrm{H}_{2}\right)=0
$$

where "b" is for "branching ratio", $\mathrm{k}^{r}$ is for "recombination rate" $\left[\mathrm{cm}^{3} \cdot \mathrm{s}^{-1}\right], \mathrm{K}^{d}$ is for "mean destruction rate" $\left[\mathrm{s}^{-1}\right]\left(\mathrm{K}^{d}=\sum_{i} k_{i}^{d} \cdot x_{i}\right.$, where $\mathrm{x}_{i}$ is the second reactant). We use indices (l,c) and exponants $(2,1)$ to distinguish between $\mathrm{C}_{3} \mathrm{H}_{2}$ and $\mathrm{C}_{3} \mathrm{H}$ in linear and cyclic forms (for example, ${ }^{2} \mathrm{~b}_{c}$ means "branching ratio for cyclic $\mathrm{C}_{3} \mathrm{H}_{2}$ "). Similar equations can be written for $l-\mathrm{C}_{3} \mathrm{H}_{2}, c-\mathrm{C}_{3} \mathrm{H}$ and $l-\mathrm{C}_{3} \mathrm{H}$. Using the observed column densities for each species at the border of the filament (i.e. row number 2), where the electron density should be the highest in our data set, and writing $\mathrm{k}_{c}^{r}=\mathrm{k}_{l}^{r}$ and $\mathrm{X} \equiv \mathrm{x}\left(\mathrm{c}-\mathrm{C}_{3} \mathrm{H}_{3}^{+}\right) / \mathrm{x}\left(1-\mathrm{C}_{3} \mathrm{H}_{3}^{+}\right)$, calculations lead to:

$$
\begin{gathered}
\mathrm{x}\left(c-\mathrm{C}_{3} \mathrm{H}_{2}\right) / \mathrm{x}\left(c-\mathrm{C}_{3} \mathrm{H}\right)=\left({ }^{2} \mathrm{~b}_{c} /{ }^{1} \mathrm{~b}_{c}\right)\left({ }^{1} \mathrm{~K}_{c}^{d} /{ }^{2} \mathrm{~K}_{c}^{d}\right) \simeq 5 ; \\
\mathrm{x}\left(l-\mathrm{C}_{3} \mathrm{H}_{2}\right) / \mathrm{x}\left(l-\mathrm{C}_{3} \mathrm{H}\right)=\left({ }^{2} \mathrm{~b}_{l} /{ }^{1} \mathrm{~b}_{l}\right)\left({ }^{1} \mathrm{~K}_{l}^{d} /{ }^{2} \mathrm{~K}_{l}^{d}\right) \simeq 2 ; \\
\mathrm{R}_{2}=\left({ }^{2} \mathrm{~b}_{c} /{ }^{2} \mathrm{~b}_{l}\right)\left({ }^{2} \mathrm{~K}_{l}^{d} /{ }^{2} \mathrm{~K}_{c}^{d}\right) \mathrm{X} \simeq 16 ; \\
\mathrm{R}_{1}=\left({ }^{1} \mathrm{~b}_{c} /{ }^{1} \mathrm{~b}_{l}\right)\left({ }^{1} \mathrm{~K}_{l}^{d} /{ }^{1} \mathrm{~K}_{c}^{d}\right) \mathrm{X} \simeq 6 .
\end{gathered}
$$

A simple and coherent set of solution for this system is ${ }^{2} \mathrm{~b}_{l} \simeq{ }^{1} \mathrm{~b}_{l} \simeq{ }^{1} \mathrm{~b}_{c} \simeq{ }^{2} \mathrm{~b}_{c} ;{ }^{1} \mathrm{~K}_{l}^{d} \simeq 2{ }^{2} \mathrm{~K}_{l}^{d}$ $;{ }^{1} \mathrm{~K}_{c}^{d} \simeq 5{ }^{2} \mathrm{~K}_{c}^{d} ;{ }^{1} \mathrm{~K}_{c}^{d} \simeq(1 / 6){ }^{1} \mathrm{~K}_{l}^{d} \mathrm{X} ;{ }^{2} \mathrm{~K}_{c}^{d} \simeq(1 / 16){ }^{2} \mathrm{~K}_{l}^{d} \mathrm{X}$.

Under the hypothesis of steady state and from the observed abundance ratio at the border of the TMC-1 filament, we find that $c-\mathrm{C}_{3} \mathrm{H}_{2}$ is destroyed approximatively 5 times slower than $c-\mathrm{C}_{3} \mathrm{H}$ and that the $l-\mathrm{C}_{3} \mathrm{H}$ mean destruction rate is twice the $l-\mathrm{C}_{3} \mathrm{H}_{2}$ one.

To our knowledge, no additional formation process of $c-\mathrm{C}_{3} \mathrm{H}_{2}$ in dark clouds can be invoked to explain an increase of its abundance with respect to $l-\mathrm{C}_{3} \mathrm{H}_{2}$. Variations of $\mathrm{R}_{2}$ (and $\mathrm{R}_{1}$ ) in TMC-1 could result from variations of the destruction rates of $c-\mathrm{C}_{3} \mathrm{H}_{2}$ and $l-\mathrm{C}_{3} \mathrm{H}_{2}$. Such an explanation has been proposed by Maluendes, McLean, \& Herbst (1993): while $c-\mathrm{C}_{3} \mathrm{H}_{2}$ is inert with respect to most neutral-neutral reactions, $l-\mathrm{C}_{3} \mathrm{H}_{2}$, as $l-\mathrm{C}_{3} \mathrm{H}$ and $c-\mathrm{C}_{3} \mathrm{H}$, are easily destroyed by these reactions. Hence, because neutral-neutral reactions proceed fast when a radical reacts with abundant atoms, it is possible in a dark cloud like TMC-1 that $R_{2}$ is one order of magnitude larger than in a more diffuse medium where the proportion of reactive ions (increasing with respect to reactive atoms) is sufficient to destroy $c-\mathrm{C}_{3} \mathrm{H}_{2}$ and $l-\mathrm{C}_{3} \mathrm{H}_{2}$ at the same rate. The same explanation holds for the progenitor ions c- $\mathrm{C}_{3} \mathrm{H}_{3}^{+}$and $\mathrm{l}_{-} \mathrm{C}_{3} \mathrm{H}_{3}^{+}$(see Cernicharo et al. 1999): while l- $\mathrm{C}_{3} \mathrm{H}_{3}^{+}$- assumed 
to be the progenitor of $l-\mathrm{C}_{3} \mathrm{H}_{2}$ - can be efficiently removed through ion-neutral reactions lowering the amount available to produce $l-\mathrm{C}_{3} \mathrm{H}_{2}, c-\mathrm{C}_{3} \mathrm{H}_{3}^{+}$is mainly affected by dissociative recombination to produce $c-\mathrm{C}_{3} \mathrm{H}_{2}$. $\mathrm{R}_{2}$ thus depends on the ion-neutral reactions of $1-\mathrm{C}_{3} \mathrm{H}_{3}^{+}$ that do not affect the cyclic ion: in dark clouds, where the abundance of reactive molecules can be hundred times larger than that of electrons, $1-\mathrm{C}_{3} \mathrm{H}_{3}^{+}$is removed faster by ion-neutral reactions than by the dissociative recombination lowering the production rate of $l-\mathrm{C}_{3} \mathrm{H}_{2}$, and increasing $\mathrm{R}_{2}$. In a more diffuse medium, where the ion-neutral reactions are much less efficient, $\mathrm{l}_{-} \mathrm{C}_{3} \mathrm{H}_{3}^{+}$and $\mathrm{c}-\mathrm{C}_{3} \mathrm{H}_{3}^{+}$are removed by the same process and $\mathrm{R}_{2}$ is then closer to ${ }^{2} \mathrm{~K}_{l}^{d} /{ }^{2} \mathrm{~K}_{c}^{d}$ (whose value also decreases with decreasing density as suggested above). This explanation is strengthened by the lower $R_{2}$ values found in the diffuse medium from absorption measurements by Cernicharo et al. (1999) - 3 to 5 versus 10 to 40 in TMC-1 and by the observed $R_{1}$ variations across the filament. We test this hypothesis in the next section.

\subsection{Chemical modelling}

In order to study the impact of physical conditions on the $R_{2}$ ratio, we have run 25 models of gas phase chemistry with different densities and visual extinctions using a time dependent chemistry code solving the system of stiff ordinary differential equations with the Gear method. Although we have used a time dependent chemistry code - samples of the evolution chemistry are shown in Fig.4 - we discuss here the results at steady state. Indeed, we would like to study the basic processes of the isomeric differentiation, and to identify the dominant reactions leading to the observed large variations of $\mathrm{R}_{2}$ in diffuse and dense clouds. We aim first at a qualitative description of the mechanism. A quantitative description would require a more accurate knowledge of the reaction rates (including branching ratios) important for $c-\mathrm{C}_{3} \mathrm{H}_{2}$ and $l-\mathrm{C}_{3} \mathrm{H}_{2}$. Fig. 4 shows that $c-\mathrm{C}_{3} \mathrm{H}_{2} / l-\mathrm{C}_{3} \mathrm{H}_{2}$ and c- $\mathrm{C}_{3} \mathrm{H}_{3}^{+} / \mathrm{l}-\mathrm{C}_{3} \mathrm{H}_{3}^{+}$abundance ratios reach values close to the steady state value, just after they reach their maximum abundance. As a first step, it is thus reasonable to consider only the processes leading to variation in isomeric abundances at steady state. We have used, as chemical network, the UMIST95 database (Millar, Farquhar, \& Willacy 1997) in a pure gas-phase scheme. As recommanded by the authors, the species $\mathrm{C}_{2} \mathrm{H}_{5}, \mathrm{C}_{2} \mathrm{H}_{6}$ and their ions have been excluded from the network. We have also excluded the species including the following atoms: $\mathrm{P}, \mathrm{Si}, \mathrm{Cl}, \mathrm{Na}, \mathrm{Mg}$. On the other hand, we have included the grain surface formation of $\mathrm{H}_{2}$ with a rate coefficient $1.5 \times 10^{-17} \mathrm{n}^{2}(\mathrm{H}) \mathrm{cm}^{-3} \mathrm{~s}^{-1}$. Moreover, we have updated the dissociative recombination rate of $\mathrm{c}_{-} \mathrm{C}_{3} \mathrm{H}_{3}^{+}$at $300 \mathrm{~K}$ to $7.0 \pm 2.0 \times 10^{-7}$ $\mathrm{cm}^{3} \mathrm{~s}^{-1}$ (Abouelaziz et al.1993) and assumed the same value for $\mathrm{l}_{-} \mathrm{C}_{3} \mathrm{H}_{3}^{+}$. Parameters of the models and initial elemental abundances are given in Table 3 and 4, respectively. 
In Fig.4, we see that $\mathrm{R}_{2}$, and also $\mathrm{X}$, increase with increasing visual extinction. This general trend of our models agrees with observations since $R_{2}$ is lower in the diffuse medium than in the TMC-1 dark cloud. The models also show that the increase is faster in higher density media. We have plotted in Fig.5 the electronic density with respect to $\mathrm{R}_{2}$ for each model. The distribution of the points exhibit clearly a correlation between the ionization fraction and the cyclic over linear ratio which can be separated in two regimes: "low" $\left(1 \leq \mathrm{A}_{V} \leq 2\right)$ and "high" $\left(5 \leq \mathrm{A}_{V} \leq 10\right), A_{V}=3 \mathrm{mag}$ being an intermediate case. In the low extinction regime, $\mathrm{R}_{2}$ strongly depends on the electronic abundance. This is an interesting result because it opens the possibility to use $\mathrm{R}_{2}$ as a tool to probe the electronic abundance in low extinction regions where $\mathrm{H}^{13} \mathrm{CO}^{+}$and $\mathrm{DCO}^{+}$cannot be detected. Why is the cyclic over linear ratio sensitive to the ionization fractionation? In the UMIST95 chemistry, $\mathrm{C}^{+}$ can destroy both $l-\mathrm{C}_{3} \mathrm{H}_{2}$ and $c-\mathrm{C}_{3} \mathrm{H}_{2}$, whereas $\mathrm{C}$ reacts only with the linear isomer. $\mathrm{R}_{2}$ is then sensitive to the electronic abundance via the $\mathrm{C}^{+} / \mathrm{C}$ ratio as shown in Fig.6. Note that the range 3 to 5 that Cernicharo et al. (1999) have observed in the diffuse medium corresponds, as expected, to high electronic abundances and to moderate visual extinctions. In the high extinction regime, $\mathrm{R}_{2}$ is independent of the $\mathrm{C}^{+} / \mathrm{C}$ ratio, while it is sensitive to the $\left(\mathrm{H}_{3}^{+}+\mathrm{C}^{+}\right) / \mathrm{O}$ ratio (Fig.7). Indeed, in the UMIST95 database the atomic oxygen reacts only with $l-\mathrm{C}_{3} \mathrm{H}_{2}\left(\mathrm{O}+l-\mathrm{C}_{3} \mathrm{H}_{2} \rightarrow \mathrm{C}_{2} \mathrm{H}_{2}+\mathrm{CO}\right)$. This reaction is negligible with respect to other destruction reactions (in particular the reactions with $\mathrm{C}^{+}$which affects both isomers) until all the carbon is locked into CO. It is thus unefficient in low visual extinction media where the abundances of $\mathrm{C}$ and $\mathrm{C}^{+}$remain high. On the other hand, it becomes the principal destruction channel of $l-\mathrm{C}_{3} \mathrm{H}_{2}$ at high visual extinctions: for $\mathrm{A}_{V}=2$ mag and a density of $10^{3} \mathrm{~cm}^{-3}$ the $\mathrm{O}+l-\mathrm{C}_{3} \mathrm{H}_{2}$ reaction is roughly $15 \%$ of the $l-\mathrm{C}_{3} \mathrm{H}_{2}$ destruction rate while it represents more than $80 \%$ of it at $\mathrm{A}_{V}=10 \mathrm{mag}$. The variations of the cyclic over linear abundance ratio of $\mathrm{C}_{3} \mathrm{H}_{2}$ can thus be understood as a consequence of the competition between neutral-neutral and ion-neutral reactions in the interstellar medium.

The determination of electronic abundances from measurements of $R_{2}$ using Fig.5 may not be straightforward. Indeed, taking into account the large uncertainties in the reaction rates and branching ratios, it is difficult to infer quantitative results from this study on the $\mathrm{C}_{3} \mathrm{H}_{2}$ isomers. We can say however that low $\mathrm{R}_{2}$ values are specific of high electronic abundance while high $\mathrm{R}_{2}$ values indicate low electronic abundance. In a more general way, isomeric ratio can be used to probe physical conditions in different media from the diffuse gas to dark clouds. In the future, cyclic and linear species other than the isomers of $\mathrm{C}_{3} \mathrm{H}_{2}$ shall be included in chemical models. $\mathrm{C}_{3} \mathrm{H}$ is a good candidate. Indeed, Kaiser et al. (1997, 1999) have shown that the neutral-neutral reaction $\mathrm{C}+\mathrm{C}_{2} \mathrm{H}_{2} \rightarrow \mathrm{C}_{3} \mathrm{H}+\mathrm{H}$ produces linear and cyclic $\mathrm{C}_{3} \mathrm{H}$, with an increase of the linear over cyclic abundance ratio with rising collision energies. It would be interesting to make measurements of $R_{1}$ in absorption in 
the diffuse medium in order to compare this neutral-neutral formation path with the $\mathrm{C}_{3} \mathrm{H}_{3}^{+}$ dissociative recombination.

This study has been partly begun. Indeed, in a recent paper, Turner, Herbst, \& Terzieva (2000) investigate the $R_{1}$ and $R_{2}$ ratios in three translucent clouds and in the dark clouds TMC-1 and L183. They compare observations to the predictions of a modified version of the New Standard Model of chemistry (Lee et al.1996) which includes cyclic and linear species for all precursor to $\mathrm{C}_{3} \mathrm{H}$ and $\mathrm{C}_{3} \mathrm{H}_{2}$. One of their conclusion is that the clear observed anticorrelation of $\mathrm{R}_{2}$ with extinction suggests that the ratios are strongly affected by formation or destruction rates, and not just by branching ratios among the relevant chemical reactions.

\section{Conclusion}

In this paper, we have presented new results about the cyclic over linear abundance ratios of $\mathrm{C}_{3} \mathrm{H}_{2}$ and $\mathrm{C}_{3} \mathrm{H}$ toward the $\mathrm{CP}$ of TMC-1. These ratios exhibit variations which are probably due to variations in the electronic abundance across the filament. Indeed, the chemical modelling of the reactions that affect $c-\mathrm{C}_{3} \mathrm{H}_{2}$ and $l-\mathrm{C}_{3} \mathrm{H}_{2}$ shows that their ratio is sensitive mainly to the behavior of the $\mathrm{C}^{+} / \mathrm{C}$ (at low visual extinction) and $\left(\mathrm{H}_{3}^{+}+\mathrm{C}^{+}\right) / \mathrm{O}$ (at high visual extinction) abundance ratios, which are both related to the electronic abundance. $\mathrm{As}_{3} \mathrm{H}_{2}$ is observed in its cyclic and linear form in different physical conditions, it could be used as a tool to probe the electronic abundance in the ISM. However, to infer accurate values of the fractional ionization from cyclic and linear isomers abundances, the branching ratios and kinetic rates used in chemical networks must be more accurately known. In addition to laboratory studies, a systematic measurement of $R_{1}$ and $R_{2}$ in different astrophysical media should be useful for this work.

Acknowledgements We thank C.M. Walmsley for useful comments and suggestions. D.F. and J.C. thank the Spanish DGCYT for fundings under grants $N^{\circ} \mathrm{PB} 96-0883$ and PNIE98-1351E. D.F. also acknownledges the Spanish Ministry of Foreign Affairs for support under grant $\mathrm{N}^{\circ} 195$. 


\section{REFERENCES}

Abouelaziz H., Gomet J.C., Pasquerault D., Rowe B.R., and Mitchell J. B. A., 1993, J. Chem. Phys. 98, 1

Adams N.G. and Smith D., 1987, ApJ 317, L25

Avery L.W. and Green S., 1989, ApJ 337, 306

Bell M.B., Feldman P.A., Watson J.K.G., McCarthy M.C., Travers M.J., Gottlieb C.A., and Thaddeus P., 1999, ApJ 518, 740

Bettens R.P.A. and Herbst E., 1996, ApJ 468, 686

Bettens R.P.A. and Herbst E., 1997, ApJ 478, 585

Cernicharo J. and Guélin M., 1987, A\&A 176, 299

Cernicharo J., Gottlieb C.A., Guélin M., Killian T.C., Paubert G., Thaddeus P., and Vrtilek J.M., 1991, ApJ 368, L39

Cernicharo J., Cox P., Fossé D., and Güsten R., 1999, A\&A 351, 341

Cox P., Walmsley C.M., and Güsten R., 1989, A\&A 209, 382

Green S., 1980, ApJ 240, 962

Green S. and Chapman S., 1978, ApJS 37, 169

Green S., Garrison B. J., Lester W. A. Jr., and Miller W. H., 1978, ApJS 37,321

Kaiser R.I., Stranges D., Lee Y.T., and Suits A.G., 1997, ApJ 477, 982

Kaiser R.I., Ochsenfeld C., Head-Gordon M., and Lee Y.T., 1999, ApJ 510, 784

Lee H.-H., Bettens R.P.A., and Herbst E., 1996, A\&AS 119, 111

Madden S.C., Irvine W. M., and Matthews H. E., 1986, ApJ 311, L27

Mangum J.G. and Wootten A., 1990, A\&A 239, 319

Maluendes S.A., McLean A.D., and Herbst E., 1993, ApJ 417, 181

Matthews H.E. and Irvine W.M., 1985, ApJ 298, L61

Millar T.J., Farquhar P.R.A., and Willacy K., 1997, A\&AS 121, 139

Millar T.J., Leung C.M., and Herbst E., 1987, A\&A 183, 109

Pratap P., Dickens J.E., Snell R.L., Miralles M.P., Bergin E.A., Irvine W.M., and Schloerb F.P., 1997, ApJ 486, 862

Sume A., Downes D., and Wilson T.L., 1975, A\&A 39, 435 
Thaddeus P., Gottlieb C.A., Hjalmarson A., Johansson L.E.B., Irvine W.M., Friberg P., and Linke R.A., 1985, ApJ 294, L49

Thaddeus P., Vrtilek J.M., and Gottlieb C.A., 1985, ApJ 299, L63

Turner B.E., Herbst E., and Terzieva R., 2000, ApJS 126, 427

Yamamoto S., Saito S., Ohishi M., Suzuki H., Ishikawa S.-I., Kaifu N., and Murakami A., 1987, ApJ 322, L55 
Table 1: Line Parameters for $\mathrm{C}_{6} \mathrm{H}, l-\mathrm{C}_{3} \mathrm{H}_{2}$ and $c-\mathrm{C}_{3} \mathrm{H}_{2}$ at Selected Positions in TMC-1

\begin{tabular}{|c|c|c|c|c|c|c|c|c|c|c|}
\hline \multirow[b]{2}{*}{$\begin{array}{l}\text { Position } \\
(", ")\end{array}$} & \multirow{2}{*}{$\begin{array}{c}\mathrm{C}_{6} \mathrm{H} \\
\mathrm{T}_{m b} \\
\mathrm{~K} \\
\end{array}$} & \multicolumn{5}{|c|}{$l-\mathrm{C}_{3} \mathrm{H}_{2}$} & \multirow[b]{2}{*}{$\begin{array}{l}\text { Position } \\
(", ",)\end{array}$} & \multicolumn{3}{|l|}{$c-\mathrm{C}_{3} \mathrm{H}_{2}$} \\
\hline & & $\begin{array}{c}\mathrm{v} L S R \\
\mathrm{~km} \mathrm{~s} \mathrm{~s}^{-1}\end{array}$ & $\begin{array}{c}\Delta \mathrm{v} \\
\mathrm{km} \mathrm{s}^{-1}\end{array}$ & $\begin{array}{c}\mathrm{T}_{m b} \\
\mathrm{~K}\end{array}$ & $\begin{array}{c}\mathrm{v}_{L S R} \\
\mathrm{~km} \mathrm{~s} \mathrm{~s}^{-1}\end{array}$ & $\begin{array}{c}\Delta \mathrm{v} \\
\mathrm{km} \mathrm{s}^{-1}\end{array}$ & & $\begin{array}{c}\mathrm{T}_{m b} \\
\mathrm{~K}\end{array}$ & $\begin{array}{c}\mathrm{v}_{L S R} \\
\mathrm{~km} \mathrm{~s} \mathrm{~s}^{-1}\end{array}$ & $\begin{array}{c}\Delta \mathrm{v} \\
\mathrm{km} \mathrm{s}^{-1}\end{array}$ \\
\hline \multirow[t]{3}{*}{0,0} & $0.41 \pm 0.02$ & $5.72 \pm 0.01$ & $0.27 \pm 0.02$ & $0.27 \pm 0.03$ & $5.83 \pm 0.02$ & $0.33 \pm 0.03$ & 0,0 & $3.69 \pm 0.59$ & $5.54 \pm 0.01$ & $0.12 \pm 0.02$ \\
\hline & $0.13 \pm 0.02$ & $6.02 \pm 0.05$ & $0.29 \pm 0.07$ & & & & & $\begin{array}{l}4.44 \pm 0.59 \\
5.46 \pm 0.59\end{array}$ & $\begin{array}{l}5.72 \pm 0.01 \\
5.89 \pm 0.02\end{array}$ & $\begin{array}{l}0.14 \pm 0.02 \\
0.20 \pm 0.02\end{array}$ \\
\hline & & & & & & & & $2.46 \pm 0.59$ & $6.12 \pm 0.02$ & $0.14 \pm 0.03$ \\
\hline$-40,0$ & $\begin{array}{l}0.31 \pm 0.03 \\
0.11 \pm 0.11\end{array}$ & $\begin{array}{l}5.74 \pm 0.01 \\
5.99 \pm 0.02\end{array}$ & $\begin{array}{l}0.25 \pm 0.00 \\
0.09 \pm 0.20\end{array}$ & $0.31 \pm 0.07$ & $5.78 \pm 0.03$ & $0.44 \pm 0.08$ & $-50,0$ & $\begin{array}{l}2.20 \pm 0.76 \\
3.99 \pm 0.76\end{array}$ & $\begin{array}{l}5.48 \pm 0.05 \\
5.70 \pm 0.03\end{array}$ & $\begin{array}{l}0.20 \pm 0.00 \\
0.20 \pm 0.00\end{array}$ \\
\hline \multirow[t]{2}{*}{0,40} & $0.29 \pm 0.04$ & $5.82 \pm 0.03$ & $0.21 \pm 0.06$ & $0.28 \pm 0.06$ & $5.85 \pm 0.02$ & $0.18 \pm 0.04$ & 0,50 & $3.96 \pm 0.71$ & $5.68 \pm 0.02$ & $0.17 \pm 0.04$ \\
\hline & $0.25 \pm 0.04$ & $6.07 \pm 0.04$ & $0.21 \pm 0.09$ & $0.26 \pm 0.06$ & $6.15 \pm 0.02$ & $0.14 \pm 0.04$ & & $4.58 \pm 0.71$ & $5.96 \pm 0.02$ & $0.28 \pm 0.05$ \\
\hline \multirow[t]{2}{*}{$40,-40$} & $0.48 \pm 0.03$ & $5.78 \pm 0.01$ & $0.25 \pm 0.00$ & $0.28 \pm 0.07$ & $5.80 \pm 0.03$ & $0.21 \pm 0.06$ & $50,-50$ & $2.83 \pm 0.78$ & $5.61 \pm 0.03$ & $0.19 \pm 0.05$ \\
\hline & $0.22 \pm 0.03$ & $6.03 \pm 0.03$ & $0.27 \pm 0.05$ & $0.23 \pm 0.07$ & $6.08 \pm 0.03$ & $0.13 \pm 0.06$ & & $4.88 \pm 0.78$ & $5.88 \pm 0.03$ & $0.34 \pm 0.06$ \\
\hline
\end{tabular}

Notes - Positions are offsets relative to the Cyanopolyyne Peak : $\alpha(1950)=04^{h} 38^{m} 38.6^{s}, \delta(1950)=25^{\circ} 35^{\prime} 45.0^{\prime \prime}$

TMC-1 exhibits two main components at $\mathrm{v}_{L S R} \simeq 5.3$ and $6.1 \mathrm{~km} \mathrm{~s}^{-1}$ (Sume, Downes, \& Wilson 1975)

When possible, we have fitted several components along a line of sight 
Table 2: Derived Column Densities for $c-\mathrm{C}_{3} \mathrm{H}$ and $l-\mathrm{C}_{3} \mathrm{H}$ in TMC-1

\begin{tabular}{cccc}
\hline $\begin{array}{c}\text { Position } \\
(", ")\end{array}$ & $\begin{array}{c}N\left(c-\mathrm{C}_{3} \mathrm{H}\right) \\
10^{12} \mathrm{~cm}^{-2}\end{array}$ & $\begin{array}{c}N\left(l-\mathrm{C}_{3} \mathrm{H}\right) \\
10^{11} \mathrm{~cm}^{-2}\end{array}$ & $\begin{array}{c}N \mathrm{c} / N \\
\left(=\mathrm{R}_{1}\right)\end{array}$ \\
\hline$-40,-40$ & $3.7 \pm 0.2$ & $\leq 4$ & $\geq 9$ \\
$-20,-20$ & $7.7 \pm 0.3$ & $8.0 \pm 0.2$ & $10 \pm 0.5$ \\
0,0 & $10.3 \pm 0.3$ & $8.4 \pm 0.1$ & $12 \pm 0.5$ \\
20,20 & $10.0 \pm 0.3$ & $7.6 \pm 0.4$ & $13 \pm 1.2$ \\
40,40 & $5.8 \pm 0.3$ & $6.7 \pm 0.5$ & $9 \pm 1.1$ \\
60,60 & $3.6 \pm 0.2$ & $6.1 \pm 0.7$ & $6 \pm 1.0$ \\
\hline $10,-70$ & $5.2 \pm 0.2$ & $7.0 \pm 0.4$ & $7 \pm 0.6$ \\
$-10,-50$ & $5.3 \pm 0.2$ & $7.8 \pm 0.2$ & $7 \pm 0.4$ \\
$-30,-30$ & $5.1 \pm 0.2$ & $8.0 \pm 0.1$ & $6 \pm 0.4$ \\
$-50,-10$ & $4.7 \pm 0.2$ & $7.1 \pm 0.5$ & $7 \pm 0.8$ \\
$-70,10$ & $3.7 \pm 0.2$ & $7.3 \pm 0.3$ & $5 \pm 0.5$ \\
\hline $30,-60^{1}$ & $7.8 \pm 0.3$ & $8.0 \pm 0.2$ & $10 \pm 0.6$ \\
$-30,0^{2}$ & $10.0 \pm 0.4$ & $8.2 \pm 0.2$ & $12 \pm 0.7$ \\
\hline Notes - Positions are offsets relative to the CP : $_{\alpha(1950)=04^{h} 38^{m} 38.6^{s}, \delta(1950)=25^{\circ} 35^{\prime} 45.0 "}$ \\
$1{ }_{c}-\mathrm{C}_{3} \mathrm{H}_{2}$ peak \\
$l-\mathrm{C}_{3} \mathrm{H}_{2}$ peak
\end{tabular}


Table 3: Parameters of the Chemical Models

\begin{tabular}{lr}
\hline Parameter & Value \\
\hline $\mathrm{n}\left(\mathrm{H}_{2}\right)\left(10^{3} \mathrm{~cm}^{-3}\right)$ & $1,3,10,30,100$ \\
$\mathrm{~T}(\mathrm{~K})$ & $1,2,3,5,10$ \\
$\mathrm{~A}_{V}(\mathrm{mag})$ & $1.3 \times 10^{-17}$ \\
$\zeta\left(\mathrm{s}^{-1}\right)$ & $2 \times 10^{-15}$ \\
$u_{i}\left(\mathrm{erg} \mathrm{cm} \mathrm{cm}^{-3}\right)$ & \\
\hline$\zeta$ is the cosmic-ray ionization rate \\
$u_{i}$ is the density of ionizing radiation at $\mathrm{A}_{V}=0$
\end{tabular}


Table 4: Initial Elemental Abundances with respect to Hydrogen

\begin{tabular}{lr}
\hline $\mathrm{He}$ & $1.4 \times 10^{-1}$ \\
$\mathrm{C}$ & $7.3 \times 10^{-5}$ \\
$\mathrm{~N}$ & $2.14 \times 10^{-5}$ \\
$\mathrm{O}$ & $1.76 \times 10^{-4}$ \\
$\mathrm{~S}$ & $2.0 \times 10^{-8}$ \\
$\mathrm{Fe}$ & $3.0 \times 10^{-9}$ \\
\hline
\end{tabular}


Figure Captions

Fig. 1. - Spectra of $\mathrm{C}_{6} \mathrm{H}$, cyclic and linear $\mathrm{C}_{3} \mathrm{H}_{2}$, and cyclic and linear $\mathrm{C}_{3} \mathrm{H}$ towards the cyanopolyyne peak of TMC-1 $\left(\mathrm{v}_{L S R}=5.8 \mathrm{kms}^{-1}\right)$. Note that the $\mathrm{C}_{6} \mathrm{H}^{2} \Pi_{3 / 2} \mathrm{~J}=15 / 2-13 / 2$ quadruplet and the $l-\mathrm{C}_{3} \mathrm{H}_{2} 1_{01}-\mathrm{O}_{00}$ transition are observed in the same bandwidth. The $\mathrm{J}=3 / 2-1 / 2 \mathrm{~F}=2-1$ and $\mathrm{F}=1-0$ transitions of $c-\mathrm{C}_{3} \mathrm{H} 2_{12}-1_{11}$ are not shown here.

Fig. 2.- Contour levels of integrated intensity in TMC-1 for: Panel (a): $\mathrm{C}_{6} \mathrm{H}^{2} \Pi_{3 / 2}$ $\mathrm{J}=15 / 2-13 / 2$ and $c-\mathrm{C}_{3} \mathrm{H}_{2} 1_{10}-1_{01}$; Panel (b): $\mathrm{C}_{6} \mathrm{H}^{2} \Pi_{3 / 2} \mathrm{~J}=15 / 2-13 / 2$ and $l-\mathrm{C}_{3} \mathrm{H}_{2} 1_{01}-0_{00}$ (the crosses indicate the measured positions); Panel (c): $\mathrm{C}_{6} \mathrm{H}^{2} \Pi_{3 / 2} \mathrm{~J}=15 / 2-13 / 2$ and $\mathrm{HC}_{3} \mathrm{~N}$ 10-9; Panel (d): $c-\mathrm{C}_{3} \mathrm{H}_{2} 1_{10}-1_{01}$ and $l-\mathrm{C}_{3} \mathrm{H}_{2} 1_{01}-0_{00}$ (numbers indicate the values of $\mathrm{R}_{2}$, i.e. the ratio $\left.N\left(c-\mathrm{C}_{3} \mathrm{H}_{2}\right) / N\left(l-\mathrm{C}_{3} \mathrm{H}_{2}\right)\right)$. All contours go from 50 to $100 \%$ of the peak value by steps of $10 \%$. The peak values are $0.16 \mathrm{~K} \cdot \mathrm{kms}^{-1}, 2.6 \mathrm{~K} \cdot \mathrm{kms}^{-1}, 0.15 \mathrm{~K} \cdot \mathrm{kms}^{-1}$, and 1.75 $\mathrm{K} \cdot \mathrm{kms}^{-1}$ for $\mathrm{C}_{6} \mathrm{H}, c-\mathrm{C}_{3} \mathrm{H}_{2}, l-\mathrm{C}_{3} \mathrm{H}_{2}$, and $\mathrm{HC}_{3} \mathrm{~N}$ respectively. The coordinates of the $(0,0)$ position are: $\alpha=04^{h} 38^{m} 38.6^{s}, \delta=25^{\circ} 35^{\prime} 45.0^{\prime \prime}$ (1950.0) corresponding to the cyanopolyyne peak. The boxes to the right compare the $\mathrm{C}_{6} \mathrm{H}$ and $l-\mathrm{C}_{3} \mathrm{H}_{2}$ spectra averaged along the six directions indicated by the arrows. Note the significant emission of $l-\mathrm{C}_{3} \mathrm{H}_{2}$ toward the west side of the TMC-1 filament where the emission of $\mathrm{C}_{6} \mathrm{H}$ decreases rapidly.

Fig. 3.- Variations of $\mathrm{R}_{2}$ (i.e. the ratio $\left.N\left(c-\mathrm{C}_{3} \mathrm{H}_{2}\right) / N\left(l-\mathrm{C}_{3} \mathrm{H}_{2}\right)\right)$ across the TMC-1 filament using: Lower panel: a constant density of $3 \times 10^{4} \mathrm{~cm}^{-3}$; Upper panel: densities derived by Pratap et al. (1997). In order to increase the signal to noise ratio, the spectra have been averaged along six rows (separated by 28") parallel to the TMC-1 filament. Lower limits in row number 6 are derived from $3 \sigma$ upper limits on the $l-\mathrm{C}_{3} \mathrm{H}_{2}$ emission. The cyanopolyyne peak $(\mathrm{CP})$ is located within row number 4.

Fig. 4.- Chemical evolution for three different visual extinctions at $\mathrm{n}\left(\mathrm{H}_{2}\right)=10^{4} \mathrm{~cm}^{-3}$ and $\mathrm{T}=10 \mathrm{~K}$. Upper boxes show abundances relative to $\mathrm{H}$ for $\mathrm{C}, l-\mathrm{C}_{3} \mathrm{H}_{2}, c-\mathrm{C}_{3} \mathrm{H}_{2}$ and $\mathrm{e}^{-}$. Lower boxes show the resulting cyclic over linear abundance ratio for $\mathrm{C}_{3} \mathrm{H}_{2}$ and $\mathrm{C}_{3} \mathrm{H}_{3}^{+}$isomers.

Fig. 5. - The predicted electronic abundance vs. $R_{2}$ at steady state. Two regimes can be distinguished according as the visual extinction is low or high.

Fig. 6. - The predicted dependence of $\mathrm{R}_{2}$ with the $\mathrm{C}^{+} / \mathrm{C}$ ratio at steady state. A clear relationship is established at low visual extinction between $R_{2}$ and the ionization fraction of atomic carbon. Note that at high visual extinctions $\mathrm{R}_{2}$ depends only weakly on $\mathrm{C}^{+} / \mathrm{C}$.

Fig. 7.- Model prediction for the relation of $\mathrm{R}_{2}$ vs. the $\left(\mathrm{H}_{3}^{+}+\mathrm{C}^{+}\right) / \mathrm{O}$ ratio at steady state. Note the good correlation between $\mathrm{R}_{2}$ and $\left(\mathrm{H}_{3}^{+}+\mathrm{C}^{+}\right) / \mathrm{O}$ at high visual extinctions. 

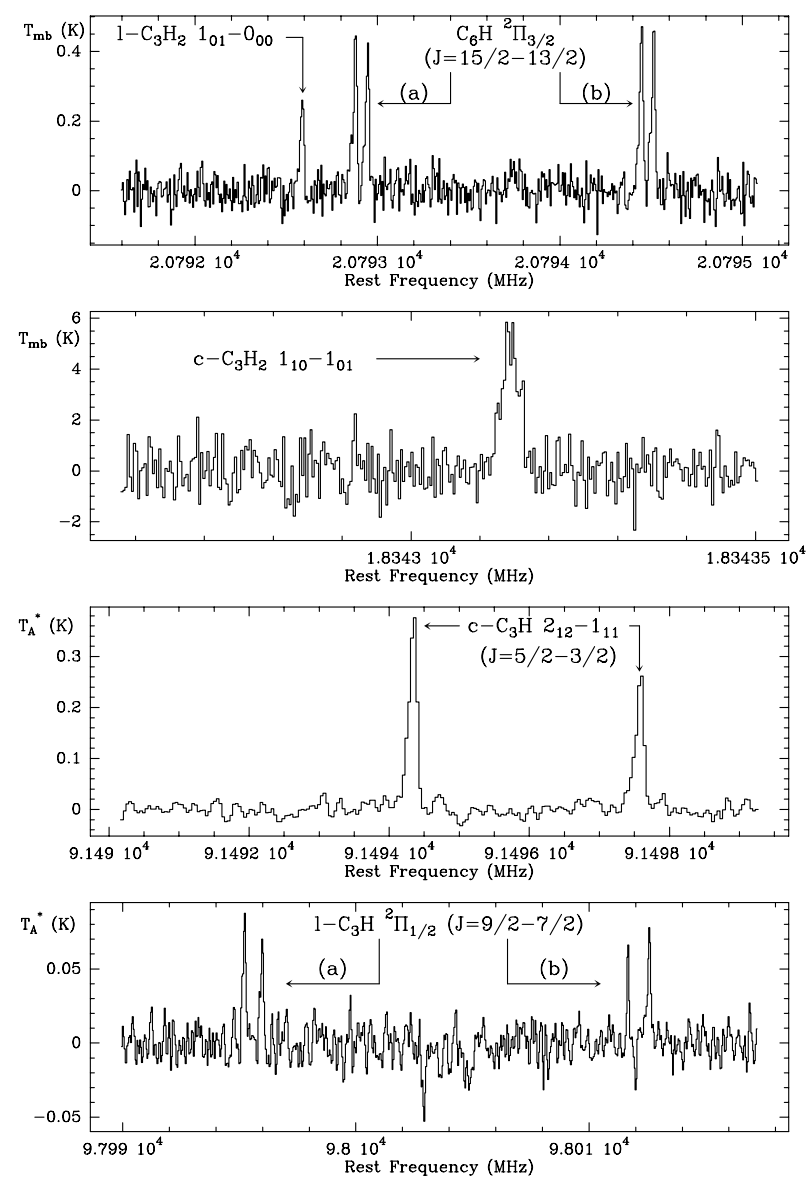


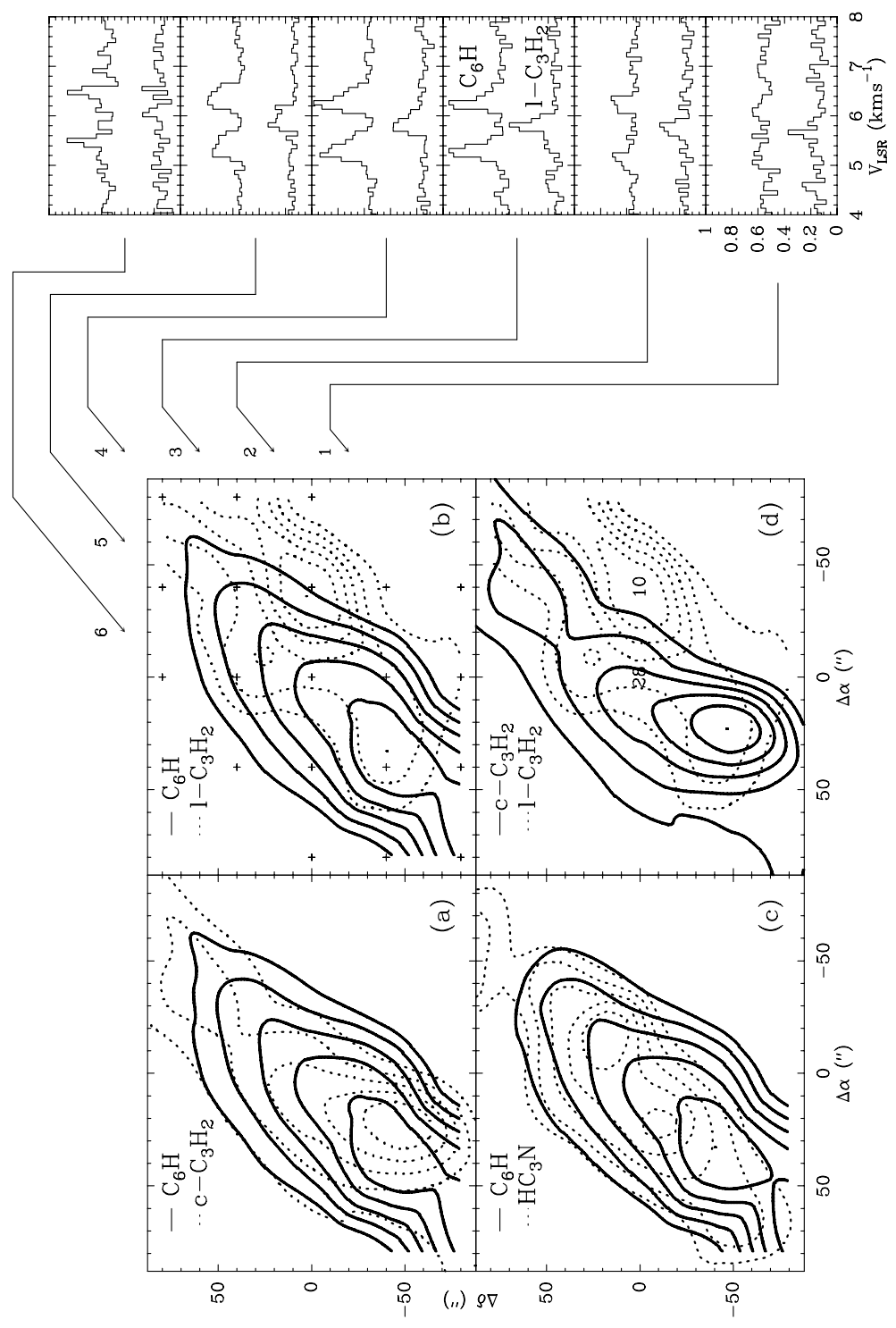



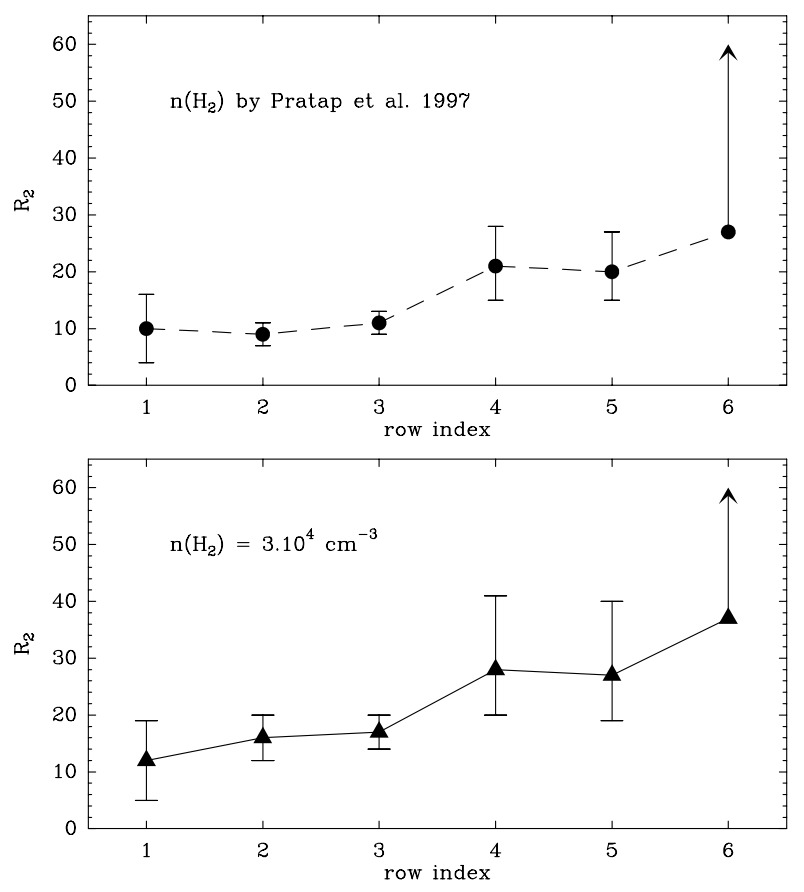

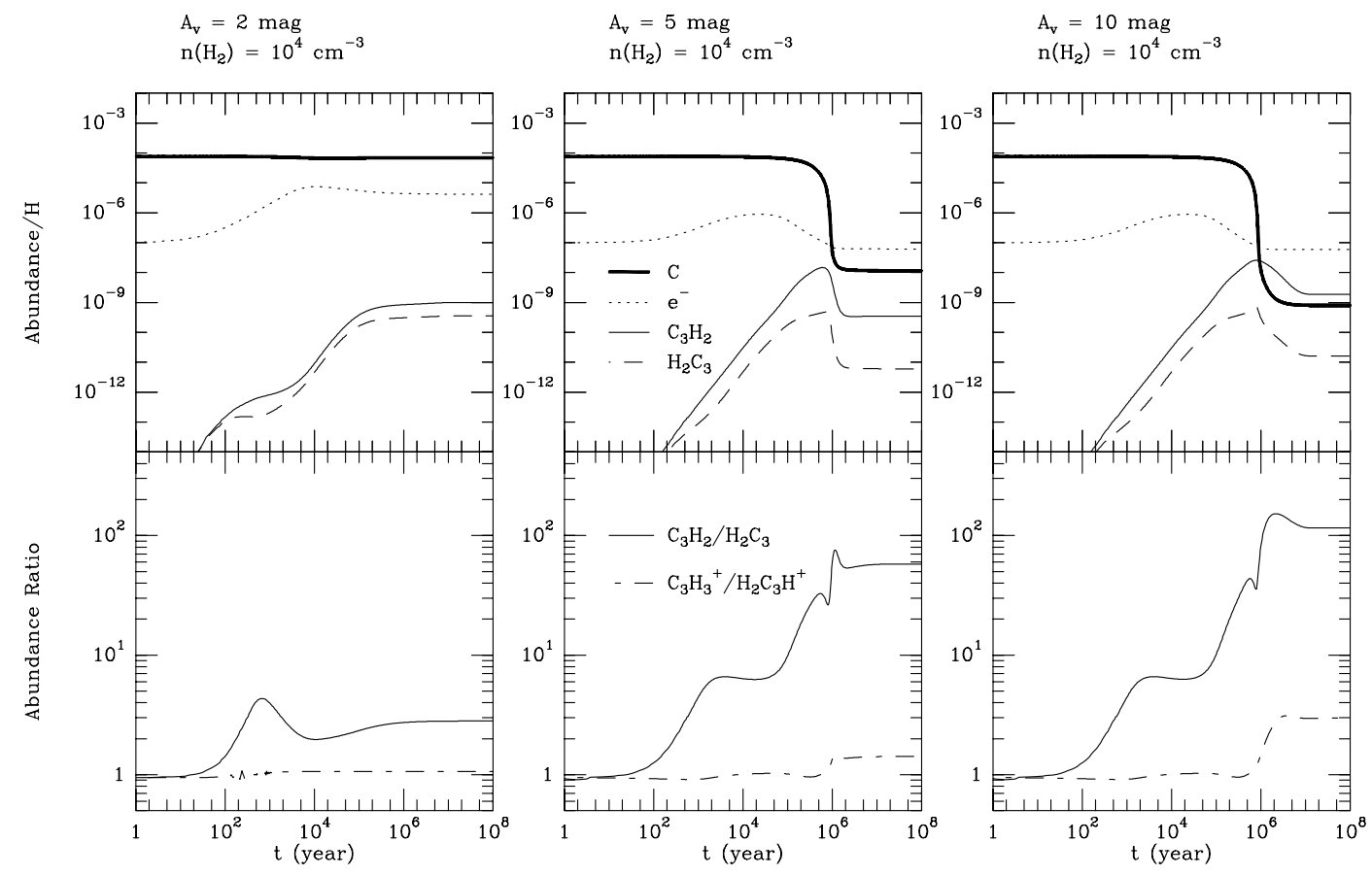


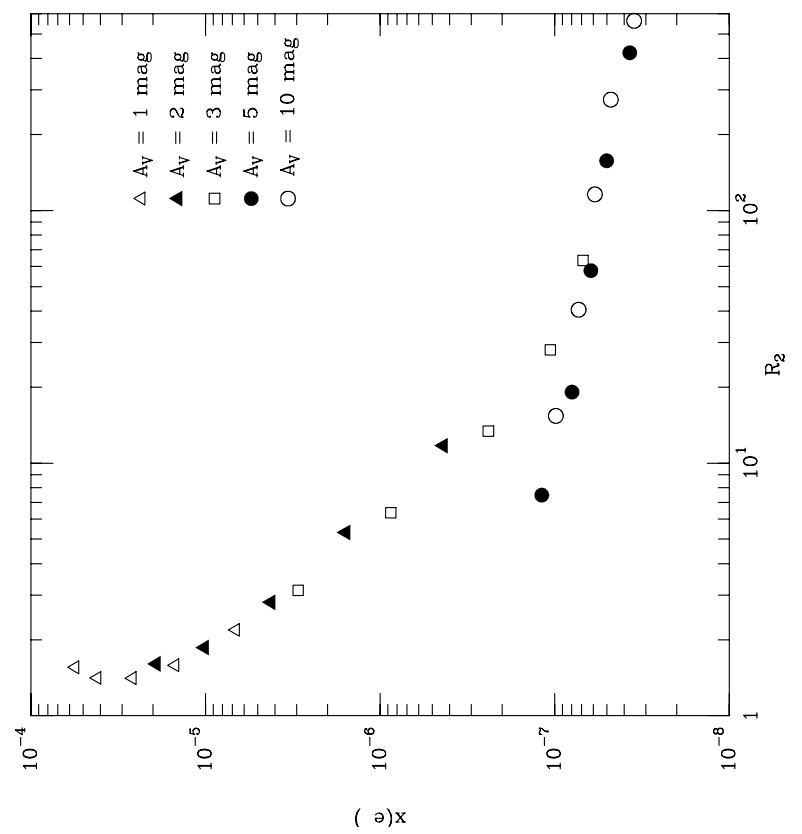




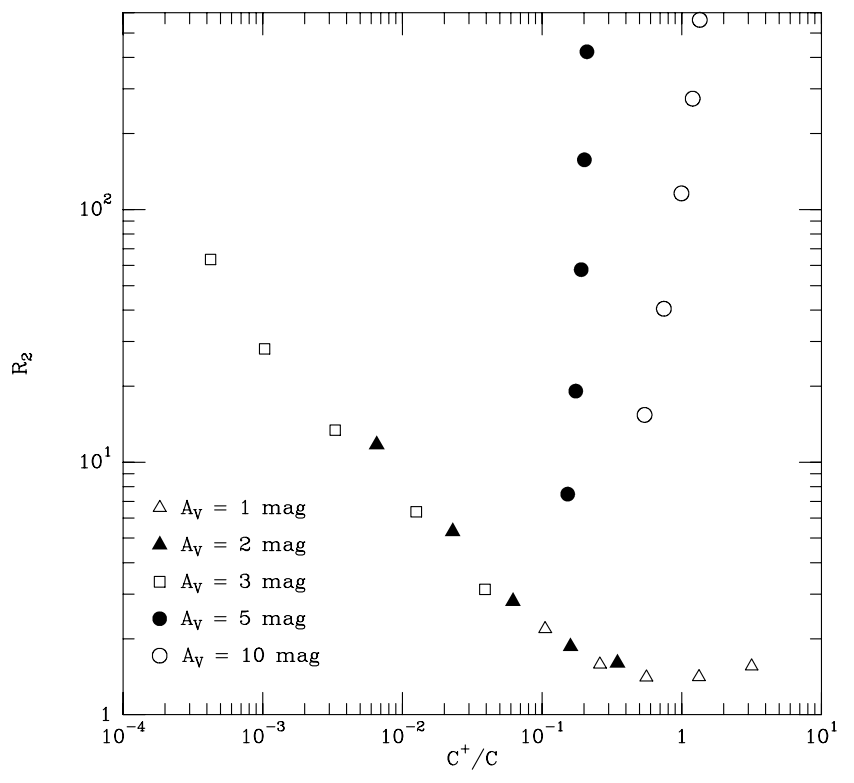




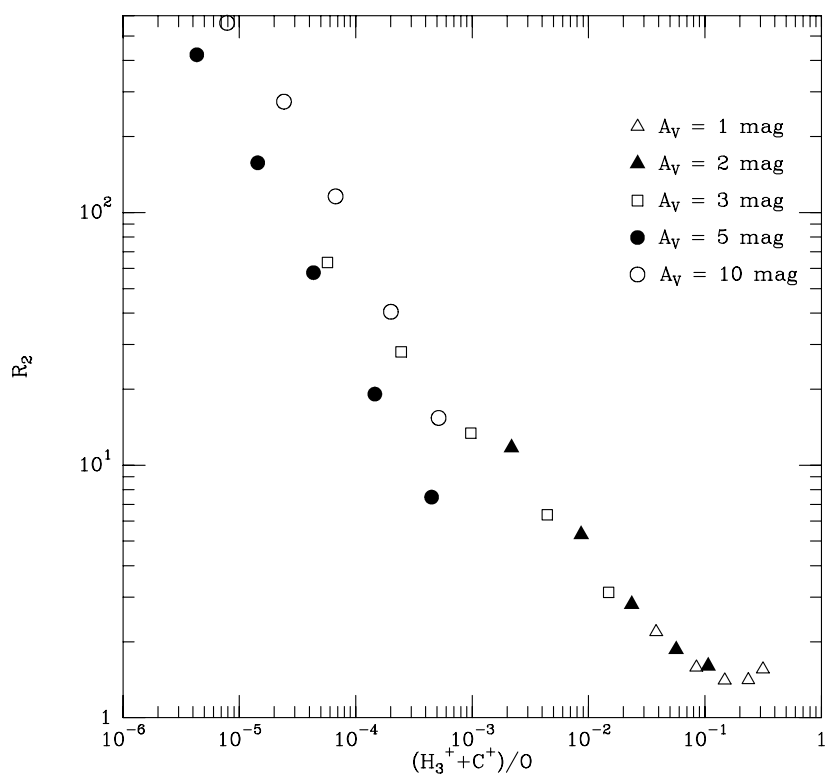

